Article

\title{
Expression of Sox2 and Oct4 and Their Clinical Significance in Human Non-Small-Cell Lung Cancer
}

\author{
Xinxin Li ${ }^{1, \dagger}$, Jinguang Wang ${ }^{2, \dagger}$, Zhiyun Xu ${ }^{1}$, Aftab Ahmad ${ }^{1}$, Encheng Li ${ }^{1}$, Yuan Wang ${ }^{1}$, \\ Suli Qin ${ }^{3}$ and Qi Wang ${ }^{1, *}$
}

1 Department of Respiratory Medicine, The Second Hospital Affiliated to Dalian Medical University, Dalian 116023, China; E-Mails: lixinxin1212@hotmail.com (X.L.); tutuxzy@163.com (Z.X.); aftab_ahmad17@hotmail.com (A.A.); doctorliencheng@163.com (E.L.); wang.yuan21@hotmail.com (Y.W.)

2 Department of Thoracic Surgery, The First Hospital Affiliated to Dalian Medical University, No. 222 Zhongshan Road, Dalian 116011, China; E-Mail: dlwangjg@hotmail.com

3 People's Military Medical Press, Beijing Fuxing Road 22 A3, Beijing 100842, China; E-Mail: qinsuli@pmmp.com.cn

$\dagger$ These authors contributed equally to this work.

* Author to whom correspondence should be addressed; E-Mail: wqdmu@yahoo.com.cn; Tel.: +86-411-86110151; Fax: +86-411-86110151.

Received: 11 April 2012; in revised form: 15 June 2012 / Accepted: 19 June 2012 / Published: 21 June 2012

\begin{abstract}
Sox2 and Oct4 are transcription factors with the characteristics of regulating self-renewal and differentiation of embryonic stem cell. The aim of this study was to detect the expression of Sox 2 and Oct4 and analyze their clinical significance in human non-small-cell lung cancer (NSCLC). Expression of Sox2 and Oct4 were assayed in cancer tissues and their corresponding paracancerous tissues from 44 patients with NSCLC and 21 patients with benign tumors using immunohistochemistry, Western blot, reverse transcription polymerase chain reaction (RT-PCR). The correlation between the expression of Sox 2 and Oct4 and tumor type, grade and prognosis and the utility of the two genes in discriminating between benign and malignant tumors were analyzed as well. The results showed that Sox 2 and Oct4 positive staining was only seen in the nuclei of cancer cells but not in either the precancerous tissues or benign tumor tissues by immunohistochemistry $(p<0.01)$. Furthermore, in the lung cancer tissue, the positive rate for Sox 2 and Oct4 was $70.5 \%$ and $54.5 \%$, respectively. Meanwhile, clinicopathological correlations showed that
\end{abstract}


the Oct4 expression level was significantly associated with poorer differentiation and higher TNM stage of the cancer $(p<0.05)$. Western blot and RT-PCR analysis showed similar results to immunohistochemistry. Follow-up analysis revealed that expression of Oct4 was significantly associated with poor prognosis of lung cancer. The conclusion is that Sox2 and Oct4 may act as the promising unit markers in directing NSCLC diagnosis and therapy. Also, Oct4 can be regarded as a novel predictor of poor prognosis for NSCLC patients undergoing resection.

Keywords: NSCLC; Sox2; Oct4; immunohistochemistry; RT-PCR; Western blot

\section{Introduction}

Lung cancer is by far one of the most common malignant tumors worldwide. It is also the leading cancer type for cancer mortality that takes account for more than $26 \%$ of all cancer deaths [1]. This is largely due to the lack of biomarkers for early diagnosis. Since the common used methods such as X-ray and sputum cancer cell assay turn out only to be useful for the diagnosis for the late stage of lung cancer, while less effective for the earlier tumor stages, which directly weaken the effect of therapy and lead to a poor prognosis. Therefore, lung cancer biomarkers have been taking a relatively important place in early diagnosis, therapy guidance and predicting prognosis $[2,3]$. Unfortunately, the currently available lung cancer biomarkers such as CEA, NSE, Cyfra21-1, CA125 are not sensitive or specific enough to be effective detection means in diagnosis, drug responses, or prognosis [4]. In view of this, more novel and effective biomarkers need to be discovered and utilized. Cancer stem cells (CSCs) are considered as a small subpopulation of cancer cells that is highly enriched with the properties of self-renewal, extensive proliferation and tumor formation [5]. Substantial and growing experimental evidence is suggesting that many cancers may be driven by this subpopulation, and it can be used as a biomarker for the diagnosis of this type of cancer. More and more CSCs have been identified and isolated in solid cancers including ovarian cancer, neuroblastoma, breast cancer and lung cancer [6,7]. Kem et al. reported that bronchoalveolar stem cells (BASCs) have the properties of self-renewal, pluripotency and proliferation, and suggested them as progenitors for lung adenocarcinoma [8].

There are many members of CSCs. Sox2 is an important member of the sox gene family. The sox genes encode transcription factors that interact with DNA through their highly conserved high mobility group (HMG) domain $[9,10]$. It is known that sox genes are expressed in a wide variety of tissues and play important roles in the regulation of organ development and cell type specification, especially in embryonic stem cell (ESC) development $[11,12]$. Sox2 is also regarded as a key factor for some cancer progress [13]. Oct4 (also known as Octamer 4) belongs to the family of POU-domain transcription factors and was first found in embryonic stem (ES) and germ cells [14]. Many studies indicated that Oct4 plays a pivotal role in maintaining the self-renewal and pluripotency of ES cells [15-17]. More recently, a growing number of cancer cells were confirmed to express Oct4, even Monk et al. found that the gene was expressed only in tumors, but not in normal somatic cells, which demonstrated that Oct4 may also be crucial in cancer development [18]. Either in ES cells or in CSCs, Sox2, together 
with Oct4, plays an important role in regulating stem cell characteristics $[17,19]$. The two genes constitute part of an important gene regulatory network and are essential for embryogenesis and the pluripotency and self-renewal of the cells. Many recent studies found that some cancers including breast carcinomas, germinomas, and pancreatic carcinomas expressed Sox2 and Oct4 simultaneously [20-22] and their expression was associated with the differentiation of the tumors [23]. All this means that the two genes appear to be significant for cancer cells' survival. For lung cancer, however, very rare reports have involved the two genes till now. In this study, we used immunohistochemistry (IHC), Western blot, and reverse transcription polymerase chain reaction (RT-PCR) to assay the expression of Sox2 and Oct4 in 44 non-small-cell lung cancer (NSCLC) mainly including squamous cell carcinoma (SCC) and adenocarcinoma patients and 21 benign pulmonary tumor patients. The correlation between the expression of Sox 2 and Oct4 and tumor type, grade, prognosis and the utility of the two genes in discriminating between benign and malignant tumors was analyzed as well.

\section{Results}

\subsection{Sox2 and Oct4 Expressions in Cancerous Tissues, Precancerous Tissues and Lung Benign} Tumor Tissue

We investigated Sox 2 and Oct4 protein expression by IHC in 44 human NSCLC cancerous and precancerous tissues and 21 human benign tumor tissues. Overall, for both Sox 2 and Oct4, only immunostaining in the nuclei of the cancer cells was thought to be positive, whereas all the precancerous tissues and benign tumor tissues showed negative staining with significant differences ( $p<0.01$, Table 1, Figure 1). Among the 44 cancerous tissues, 31 showed positive staining for Sox 2 with a positive rate of $70.5 \%$, and 24 showed positive staining for Oct4 with a positive rate of $54.5 \%$. Meanwhile, 16 cases showed both Sox2 and Oct4 positive staining, with a co-positive rate of $36.4 \%$ (16/44); and 39 cases showed either Sox2 or Oct4 positive staining, with a co-positive rate as high as $88.6 \%$.

In order to confirm the IHC results, Western blot and RT-PCR were performed. On the whole, both the Western blot and RT-PCR analysis results were in line with the IHC results. That is the expression of the two factors of Sox 2 and Oct4 in NSCLC tissues was significantly higher than that of their paracancerous tissues and the benign tumors at both the protein and mRNA level (Figure 2). Nevertheless, small differences were still seen. For example, some cases with negative expression of Sox2 and Oct4 by IHC contrarily showed low-expression of them in Western blotting or RT-PCR, as shown in Figure 2. This may be due to the rough judgment of IHC.

Table 1. Sox2 and Oct4 expressions in cancerous tissues, precancerous tissues and benign lung tumor tissue.

\begin{tabular}{|c|c|c|c|c|c|c|c|c|c|}
\hline & \multicolumn{3}{|c|}{ Cancerous tissues } & \multicolumn{3}{|c|}{ Paracancerous tissues } & \multicolumn{3}{|c|}{ Benign tumor tissues } \\
\hline & ${ }^{\mathrm{a}}$ Pos & ${ }^{\mathbf{b}} \mathrm{Neg}$ & Totle & Pos & Neg & Totle & Pos & Neg & Totle \\
\hline Sox 2 & $31(70.5 \%)$ & 13 & 44 & 0 & 44 & 44 & 0 & 21 & 21 \\
\hline Oct4 & $24(54.5 \%)$ & 20 & 44 & 0 & 44 & 44 & 0 & 21 & 21 \\
\hline
\end{tabular}


Figure 1. Representative expression patterns of Sox2 (A-C) and Oct4 (D-F) in cancerous tissues and their corresponding paracancerous tissues. Brown grains represent a positive signal. The positive expression site of Sox 2 and Oct4 was mainly localized in the nucleus of tumor cells. Sox2 positive expression in lung squamous cell carcinoma (SCC) (A) and lung adenocarcinoma (B); Sox2 expression negative in paracancerous tissue (C); Oct4 staining positive in lung SCC (D) and lung adenocarcinoma (E); Oct4 negative expression in paracancerous tissue $(\mathbf{F})$.


Figure 2. Western blot and reverse transcription polymerase chain reaction (RT-PCR) analysis on the expression of Sox 2 and Oct4 in lung SCC, adenocarcinoma, paracancerous and benign tumor tissues; (A) Western blot; (B) RT-PCR. ${ }^{\text {a }}$ SCC = squamous cell carcinoma; ${ }^{\mathrm{b}}$ para $=$ precancerous; ${ }^{\mathrm{c}} \mathrm{ad}=$ adenocarcinoma .

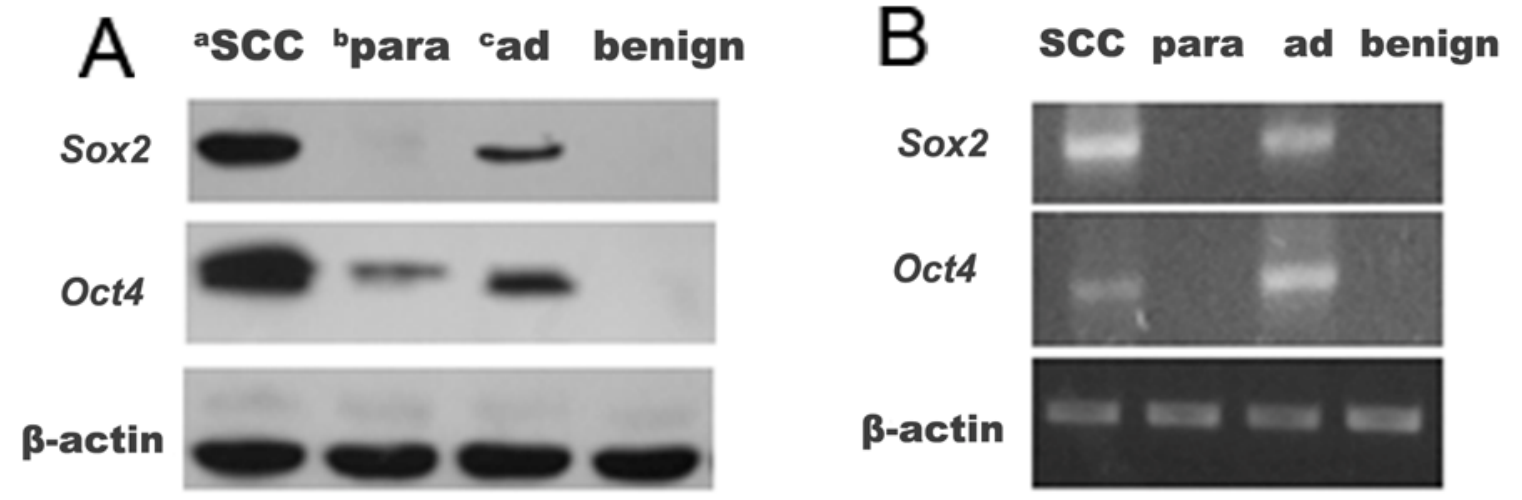




\subsection{Clinicopathological Correlations}

The association between Sox 2 and Oct4 expression levels and the clinicopathological characteristics of the lung cancer patients is summarized in Table 2. Neither Sox 2 nor Oct4 expression was correlated to age, sex, tumor location and pathological type. However, higher levels of Oct4 expression were significantly associated with poorer differentiation and higher TNM stage of the cancer $(p<0.05)$, whereas Sox2 did not have this correlation.

Table 2. Correlation of Sox 2 and Oct4 expressions to clinicopathological features of non-small-cell lung cancer (NSCLCs).

\begin{tabular}{|c|c|c|c|c|c|c|c|}
\hline \multirow{2}{*}{ Variables } & \multirow{2}{*}{ Cases } & \multicolumn{3}{|c|}{ Sox2 } & \multicolumn{3}{|c|}{ Oct4 } \\
\hline & & ${ }^{\mathbf{a}}$ Neg & ${ }^{\mathbf{b}}$ Pos & $p$ & Neg & Pos & $p$ \\
\hline Age (year) & & & & 0.831 & & & 0.614 \\
\hline$<60$ & 18 & 5 & 13 & & 9 & 9 & \\
\hline$\geq 60$ & 26 & 8 & 18 & & 11 & 15 & \\
\hline Gender & & & & 0.340 & & & 0.757 \\
\hline Male & 33 & 11 & 22 & & 15 & 17 & \\
\hline Female & 11 & 2 & 9 & & 5 & 7 & \\
\hline Location & & & & 0.469 & & & 0.507 \\
\hline Right & 24 & 6 & 18 & & 12 & 12 & \\
\hline Left & 20 & 7 & 13 & & 8 & 12 & \\
\hline Differentiation & & & & 0.755 & & & $0.009 *$ \\
\hline Well & 14 & 5 & 9 & & 10 & 4 & \\
\hline Moderately & 13 & 4 & 9 & & 7 & 6 & \\
\hline Poorly & 17 & 4 & 13 & & 3 & 14 & \\
\hline TNM stage & & & & 0.318 & & & $0.015 *$ \\
\hline $\mathrm{I} \sim \mathrm{II}$ & 29 & 10 & 19 & & 17 & 12 & \\
\hline III $\sim \mathrm{IV}$ & 15 & 3 & 12 & & 3 & 12 & \\
\hline Pathological type & & & & 0.599 & & & 0.378 \\
\hline Adnocarcinoma & 23 & 6 & 17 & & 9 & 14 & \\
\hline SCC & 21 & 7 & 14 & & 11 & 10 & \\
\hline
\end{tabular}

\subsection{Correlation between Sox2 and Oct4 Expression}

These two transcription factor expressions can be observed co-localized in the same areas of the cell nuclei in some malignant tumors with around $36.4 \%$ of the cases showing both Sox 2 and Oct 4 positive staining and around $88.6 \%$ of the cases showing either Sox 2 or Oct4 positive staining, indicating that the joint detection of the two factors is helpful. However, their expressions were also independently heterogeneous: High expression of Sox2 (grade 3) always lead to low expression (grade 2) or even negative detection of Oct4 (data not shown). In order to know whether the two factors Sox2 and Oct4 correlated statistically, a cross analysis was performed, and the results showed that their expressions were not significantly correlated $(p>0.05$, Table 3$)$. 
Table 3. Sox2 and Oct4 expression cross tabulation.

\begin{tabular}{cccc}
\hline & \multicolumn{2}{c}{ Oct4 } & Total \\
\cline { 2 - 3 } Sox2 & negative & positive & \\
\hline negative & 5 & 8 & 13 \\
positive & 15 & 16 & 31 \\
Total & 20 & 24 & 44 \\
\hline
\end{tabular}

\subsection{Relation to Survival}

Overall survival was measured for the NSCLC patients from the onset of treatment to the date of death or the survival status at the last date of the one-year follow-up. During the one-year follow-up period, 16 patients were found with recurrence or metastases, 19 patients died of cancer-related causes. A multivariate survival analysis was performed with a Cox regression model for each predictor of prognosis of IHC results. The results showed that patients with positive Oct4 immunostains had an overall poorer survival compared to the patients with negative stained tumors $(p=0.008$, Figure 3$)$. However, there was no significant correlation between Sox2 immunostaining positive and survival for the NSCLC patients ( $p>0.05$, Figure 3).

Figure 3. Kaplan-Meier curves for overall survival rates according to Sox2 expression status (A) and Oct4 expression status (B). Positive expression of Oct4 was significantly associated with poor overall survival $(p<0.05)$.
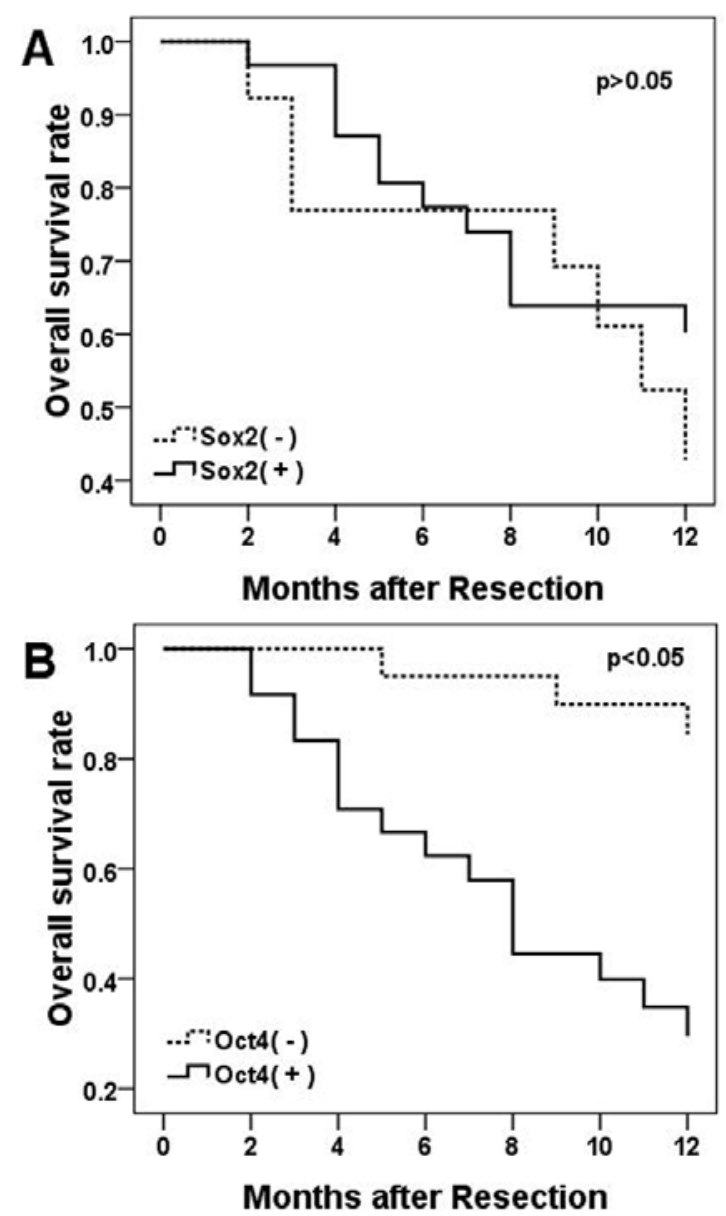


\section{Discussion}

In this study, we investigated the expression of Sox 2 and Oct4 in 44 human NSCLC cancerous and their precancerous tissues and 21 benign human tumor tissues. The present investigation revealed several novel observations. First, the results showed that there were $70.5 \%$ and $54.5 \%$ positive expressions of Sox2 and Oct4, respectively, for NSCLC. However, all of their corresponding paracancerous tissues and the benign tumor tissues were negative for these two transcription factors. All this indicates that Sox 2 and Oct4 can be potential novel biomarkers to distinguish cancer from noncancerous lesions and benign lung tumors. Second, Oct4 but not Sox2 expression level was significantly associated with poorer differentiation and higher TNM stage of the tumors and poor clinical outcome of the patients $(p<0.05)$. Moreover, neither Sox 2 nor Oct4 expression was correlated to age, sex, tumor location, or histological type of the cancers. Third, each of Sox 2 and Oct 4 alone had some limitation of sensitivity as a biomarker of NSCLC, however, when combined, the sensitivity was significantly improved to $88.6 \%$, which suggests that Sox 2 and Oct4 can be regarded as coalition predictive factors for patients with lung adenocarcinoma or SCC, and the significance of co-detection of the two factors was much more sensitive than detection of one factor alone.

Since the understanding of lung cancer tumor genesis and development may provide the possibility of its therapy or even prevention, the CSCs theory might be one of the ideal models to achieve this aim. As crucial transcription factors that maintain embryonic stem cell differentiation and pluripotency, Sox 2 and Oct4 have also been recognized as having "stemness" characteristics in cancer cells in more recent research, therefore, Sox2 and Oct4 may lead to cells immortality, self-renewable and invasive properties of cancer cells [13-19]. Supporting this hypothesis, it was suggested that knocking down Sox2 and Oct4 in tumor-initiating cells would lead to the loss of the self-renewal, proliferating and tumorigenic capacities and result in CSC-like cell apoptosis of cancer cells [24,25].

Adenocarcinoma and SCC are the most frequent and aggressive lung cancer types of NSCLC and previous studies have pointed out that stem cells must be involved in their development and maintenance. Bronchoalveolar stem cells (BASCs), which were capable of multipotent differentiation and self-renewal in both normal lung and lung cancer, are involved in the process normal cells of the distal lung developing into adenocarcinoma [26]. In other words, BASCs can be seen as the putative cells of origin for this subtype of lung cancer. Some reports shown that Oct4 is exhibited in BASCs [8]. Our study suggests that not only Oct4 but also Sox 2 may play an impotent role in this procedure of lung cancer tumorigenesis.

For SCC, the cancer came from bronchial epithelium and commonly arose near a central bronchus. Loss of the $3 p$ arm and amplification of the 3q25-27 region were identified in most of the NSCLC cases, these genomic imbalances seem to contribute to the pathogenesis of lung cancer, especially the product of $3 \mathrm{q} 26$ is potentially involved in the control of cell proliferation and malignant transformation [27]. Further investigations have indicated that 3q26.3 aberrations, in which Sox2 acts as a driver gene, were proposed as an oncogene in lung SCC and Sox2 actions along the carcinomagenesis sequence [28-30]. Our work supported that Sox2 might be a promising target for lung SCC therapy, as well as a director to lung SCC. And because of the absence of Sox2 in benign lung tumors and normal lung tissues, this target therapy might have less side effects. Whether Oct4 plays a similar role needs to be studied further. For adenocarcinoma, no relevant studies have been 
reported yet, but our research revealed no significant difference for the expression of Sox2 and Oct4 between SCC and adenocarcinoma. So suggest the hypothesis that the two transcription factors play an important role in the adenocarcinoma neoplastic processes as well. More research needs to be carried out to clarify the mechanism.

Although Sox2 and Oct4 expressions performed independently in some of the cases, they were more often co-located in the same area. Possible linkages between them in lung cancer are considered, such as the Sox2-Oct4 complex. It was revealed that they co-occupy the promoters of several hundred target genes and also form core transcription regulatory circuitry together [19]. Several studies have suggested that the Sox2-Oct4 complex is essential for the transforming growth factor- $\beta$ (TGF- $\beta$ ) and wnt $/ \beta$-catenin pathways, which have emerged as other important players in the self-renewal and maintenance of cancer stem cells [31].

Interestingly, our results showed that only $36.4 \%$ of all samples co-expressed Sox 2 and Oct 4 and that their expression levels were separate, or even reversed. This finding raised a new puzzling question: What is the relationship between Sox2 and Oct4, cooperative or interactive? In human ESCs, Sox2 and Oct4 are thought to be regulated by autoregulatory loops, and over-expression of Sox2 caused a decrease in Oct4 [31,32]. Bernadt et al. demonstrated that over-expression of Sox2, but not Oct4, inhibited the activity of Oct4 promoter in ES cells [33]. Although this finding was mainly observed in ES cells, lung SCC appears to be the likely trend in our work: The Sox2 over-expression always lead to Oct4 under-expression or non-expression. We have reasonable ground to propose that in lung SCC, the Sox2 and Oct4 regulation network also works to co-effect the cancer stem cell differentiated and self-renewal. However, the mechanisms involved are far from clear, and more work should be performed. What we confirmed is that the combinatorial analysis of Sox2 and Oct4 expression in NSCLCs is more helpful than the single analysis.

The current results demonstrate that Sox 2 and Oct4 can be detected in human lung cancer by IHC, Western blot and RT-PCR. However, there are some differences among them that can hardly be ignored, for example, some cases display expression of the two factors with Western blot and RT-PCR, while they cannot be observed when staining with IHC in cancer cell. The reason might be that Sox2 and Oct4 mainly express in cancer cells, but a small amount of other cells such as leukomonocytes or interstitial cells can also express these and hence be detected with these tests. It also may be due to the rough judgment of IHC. However, IHC is more visualized and veracious for clinical samples.

Other studies have also shown that Sox 2 or Oct4 over-expression is associated with poor prognosis in diverse cancers $[13,34]$. However, to our knowledge, there is little information about the prognostic value of the two factors in human NSCLC. Our research found that a positive expression of Oct4 protein and mRNA were independent prognostic factors for a poor overall survival of lung cancer patients.

The present study indicated that Oct4, but not Sox2, was a novel marker in predicting the prognosis of NSCLC. To further confirm the significance of the two factors in the diagnosis and therapy of NSCLC, our group is now working on an easy-test of Sox2 and Oct4 and a quick knocking down of the two transcription factors. 


\section{Experimental Section}

\subsection{Specimens and Clinical Pathological Data}

This study was approved by the Institutional Review Board and Human Ethic of the First affiliated hospital of Dalian Medical University and the Second affiliated hospital of Dalian Medical University. Forty-four NSCLC patients and 21 pulmonary benign tumor patients who underwent surgical treatment in the First or the Second affiliated hospital of Dalian Medical University during the period from 2008 to 2011 were collected. All the patients were diagnosed with pathologic identification. Among them, 45 were men and 20 were women, aging from 37 to 78 years of age with a mean age of 62.6. The 44 lung cancers included 23 adenocarcinoma and 21 SCCs; 21 benign tumor included six tuberculosis and 15 inflammatory disease cases. The describing data of the 65 patients are listed in Table 2. Surgically removed specimens of fresh lung cancer tissues, their paracancerous tissues and benign tumor tissues were partly fixed in buffered formalin and embedded in paraffin blocks for IHC analysis and the rest of the samples were stored in the refrigerator at $-80{ }^{\circ} \mathrm{C}$ for RT-PCR and Western blot.

\subsection{Imunohistochemical Analysis}

Immunohistochemical stains were performed on formalin-fixed, paraffin-embedded $5 \mu \mathrm{m}$ sections using the SP-9000 Histostain tm-plus kits (ZYMED, USA). The sections were deparaffinized in xyliene, and microwaved in antigen retrieval buffer for $15 \mathrm{~min}$. Endogenous peroxidase activity was blocked by incubation in 3\% $\mathrm{H}_{2} \mathrm{O}_{2}$ solution in methanol for $10 \mathrm{~min}$. Slides were blocked in $10 \%$ goat serum in TBS for $15 \mathrm{~min}$, and then monoclonal mouse anti-Sox 2 or anti-Oct 4 antibody (Sigma, USA) both diluted at 1:100 was added for incubation for $4{ }^{\circ} \mathrm{C}$ overnight. Specimens were stained with 3',3-diaminobenzidine tetrahydrochloride (DAB), counterstained with hematoxylin, dehydrated and mounted in Diatex. A PBS-only staining sample was used as a background control, gastric cancer and seminoma tissues were used as positive controls for Sox2 and Oct4, respectively.

Only nuclear staining was considered as positive. Sox2 and Oct4 expression were defined as any of the following four patterns and scored according to the extent of positivity of the stained cells: 0 , no positive cells seen; 1 , around $<10 \%$ of cells stained; 2 , around $10 \%-50 \%$ of cells stained; and 3 , around $>50 \%$ of cells stained; for statistical analyses, the grades 0 and 1 were considered as negative, the grades 2 and 3 were considered as positive. The details were described in our previous work [13].

\section{3. $R T-P C R$}

Total RNA was isolated from the fresh tissue samples of lung cancer tissues, their paracancerous tissues and benign tumor tissues using Trizol reagent (Takara, China) according to the manufacturer's protocol. Extracted RNA was dissolved in RNA free water. cDNA was synthesized with the RNA PCR kit (Takara, China). RT-PCR for Sox2, Oct4 and $\beta$-actin was performed using the primer sequences given in Table 4. PCR products were analyzed by electrophoresis of $10 \mu \mathrm{L}$ of each PCR reaction mixture in a $1.5 \%$ agarose gel. 
Table 4. Primers for Sox2, Oct4 and $\beta$-actin genes.

\begin{tabular}{llll}
\hline $\begin{array}{l}\text { Gene } \\
\text { name }\end{array}$ & Primer sequence & Size (bp) & $\begin{array}{l}\text { Annealing } \\
\text { temperature }\left({ }^{\circ} \mathrm{C}\right)\end{array}$ \\
\hline Sox2 & $\begin{array}{l}\text { F cccceggcggcaatagca } \\
\text { R tcggcgccggggagatacat }\end{array}$ & 448 & 58 \\
Oct4 & $\begin{array}{l}\text { F tccttcgca agccetcat } \\
\text { R tgacggtgcagggctccggggaggccc catc }\end{array}$ & 408 & 55 \\
R-actin & $\begin{array}{l}\text { F gatcttgatcttcattgtgctggg } \\
\text { R tcgtcaccaactgggacgacatgg }\end{array}$ & 752 & 55 \\
\hline
\end{tabular}

\subsection{Western Blot Analysis}

Proteins isolated from the fresh tissue samples of lung cancerous tissues, their paracancerous tissues and benign tumor tissues using PARA kit (Beyotime, China) according to the manufacturer's protocol. $10 \mu \mathrm{L}$ of the protein samples were separated on a 10\% SDS-PAGE gel (Bio-Rad) for $120 \mathrm{~min}$ at $200 \mathrm{~mA}$, and then transferred to nitrocellulose membrane (Whatman, Kent, UK). After blocking in 5\% fat-free milk, the membrane was incubated with the dilution of the anti-Sox2 antibody (1:100) and anti-Oct4 antidody (1:100), respectively, overnight at $4{ }^{\circ} \mathrm{C}$ and the dilution of the secondary IgG-horseradish peroxidase (HRP) conjugated antibody for $1 \mathrm{~h}$ at room temperature. The stained membranes were visualized by enhanced chemiluminescence reaction using the ECL Plus (GE Healthcare, USA).

\subsection{Follow-Up}

Follow-up information was available for the NSCLC patients for a period of minimum 1 year. All patients were monitored prospectively by physical examination, X-ray or ultrasonography every 1-3 months thereafter for surveillance of recurrence or metastases. The recurrence, metastases or death times were recorded.

\subsection{Statistical Analysis}

SPSS 17.0 was employed to analyze all data obtained in this study. The pearson chi-square was used for positive staining rates comparison between subgroups. The bands of Western blot and RT-PCR were evaluated using the $t$-test. A multivariate survival analysis was performed with a Cox regression model for each predictor of prognosis, as well as $95 \%$ confidence intervals. Overall survival was assessed by Kaplan-Meier analysis. Only $p<0.05$ was considered as statistical significance.

\section{Conclusions}

In summary, transcription factors Sox2 and Oct4 were expressed and localized in the nucleus of the wide majority of lung SCC and adenocarcinoma, but not in their paracancerous tissues and benign tumor tissues, suggesting that Sox2 and Oct4 can act as novel unite markers and ideal therapeutic targets. The expression of Oct4 enables the tumors to have a higher degree of stemness tumor cells, which in turn results in poorer clinical outcome for patients with NSCLCs. 


\section{Acknowledgments}

The authors are grateful to Z. Suo for assistance. This work was supported by the National Nature Science Foundation of China (81071228) and Education Foundation of Liaoning Province (200904375).

\section{References}

1. Jemal, A.; Siegel, R.; Xu, J.; Ward, E. Cancer statistics, 2010. CA Cancer J Clin. 2010, 60, 277-300.

2. Leget, G.A.; Czuczman, M.S. Use of rituximab, the new FDA-approved antibody. Curr. Opin. Oncol. 1998, 10, 548-551.

3. Marrero, J.A.; Lok, A.S. Newer markers for hepatocellular carcinoma. Gastroenterology 2004, 127, S113-S119.

4. Tarro, G.; Perna, A.; Esposito, C. Early diagnosis of lung cancer by detection of tumor liberated protein. J. Cell Physiol. 2005, 203, 1-5.

5. Rey, T.; Morrison, S.J.; Clarke, M.F.; Weissman, I.L. Stem cells, cancer, and cancer stem cells. Nature 2001, 414, 105-111.

6. Jordan, C.T.; Guzman, M.L.; Noble, M. Cancer stem cells. N. Engl. J. Med. 2006, 355, 1253-1261.

7. Al-Hajj, M. From the cover: Prospective identification of tumorigenic breast cancer cells. Proc. Natl. Acad. Sci. USA 2003, 100, 3983-3988.

8. Kim, C.F.B.; Jackson, E.L.; Woolfenden, A.E.; Lawrence, S.; Babar, I.; Vogel, S.; Crowley, D.; Bronson, R.T.; Jacks, T. Identification of bronchioalveolar stem cells in normal lung and lung cancer. Cell 2005, 121, 823-835.

9. Ferrari, S.; Harley, V.R.; Pontiggia, A.; Goodfellow, P.N.; Lovell-Badge, R.; Bianchi, M.E. Sry, like hmg1, recognizes sharp angles in DNA. EMBO J. 1992, 11, 4497-4506.

10. Weiss, M.A. Floppy sox: Mutual induced fit in hmg (high-mobility group) box-DNA recognition. Mol. Endocrinol. 2001, 15, 353-362.

11. Pevny, L.H.; Lovell-Badge, R. Sox genes find their feet. Curr. Opin. Genet. Dev. 1997, 7, 338-344.

12. Wegner, M. From head to toes: The multiple facets of sox proteins. Nucleic Acids. Res. 1999, 27, 1409-1420.

13. Wang, Q.; He, W.; Lu, C.; Wang, Z.; Wang, J.; Giercksky, K.E.; Nesland, J.M.; Suo, Z. Oct3/4 and sox 2 are significantly associated with an unfavorable clinical outcome in human esophageal squamous cell carcinoma. Anticancer Res. 2009, 29, 1233-1241.

14. Scholer, H.R.; Hatzopoulos, A.K.; Balling, R.; Suzuki, N.; Gruss, P. A family of octamer-specific proteins present during mouse embryogenesis: Evidence for germline-specific expression of an oct factor. EMBO J. 1989, 8, 2543-2550.

15. Nichols, J.; Zevnik, B.; Anastassiadis, K.; Niwa, H.; Klewe-Nebenius, D.; Chambers, I.; Scholer, H.; Smith, A. Formation of pluripotent stem cells in the mammalian embryo depends on the pou transcription factor oct4. Cell 1998, 95, 379-391. 
16. Hay, D.C.; Sutherland, L.; Clark, J.; Burdon, T. Oct-4 knockdown induces similar patterns of endoderm and trophoblast differentiation markers in human and mouse embryonic stem cells. Stem Cells 2004, 22, 225-235.

17. Boiani, M.; Scholer, H.R. Regulatory networks in embryo-derived pluripotent stem cells. Nat. Rev. Mol. Cell Biol. 2005, 6, 872-884.

18. Monk, M.; Holding, C. Human embryonic genes re-expressed in cancer cells. Oncogene 2001, 20, 8085-8091.

19. Boyer, L.A.; Lee, T.I.; Cole, M.F.; Johnstone, S.E.; Levine, S.S.; Zucker, J.P.; Guenther, M.G.; Kumar, R.M.; Murray, H.L.; Jenner, R.G.; et al. Core transcriptional regulatory circuitry in human embryonic stem cells. Cell 2005, 122, 947-956.

20. Rodriguez-Pinilla, S.M.; Sarrio, D.; Moreno-Bueno, G.; Rodriguez-Gil, Y.; Martinez, M.A.; Hernandez, L.; Hardisson, D.; Reis-Filho, J.S.; Palacios, J. Sox2: A possible driver of the basal-like phenotype in sporadic breast cancer. Mod. Pathol. 2007, 20, 474-481.

21. Hattab, E.M.; Tu, P.H.; Wilson, J.D.; Cheng, L. Oct4 immunohistochemistry is superior to placental alkaline phosphatase (plap) in the diagnosis of central nervous system germinoma. Am. J. Surg. Pathol. 2005, 29, 368-371.

22. Sanada, Y.; Yoshida, K.; Ohara, M.; Oeda, M.; Konishi, K.; Tsutani, Y. Histopathologic evaluation of stepwise progression of pancreatic carcinoma with immunohistochemical analysis of gastric epithelial transcription factor sox2: Comparison of expression patterns between invasive components and cancerous or nonneoplastic intraductal components. Pancreas 2006, 32, 164-170.

23. Till, J.E. Stem cells in differentiation and neoplasia. J. Cell Physiol. Suppl. 1982, 1, 3-11.

24. Gangemi, R.M.; Griffero, F.; Marubbi, D.; Perera, M.; Capra, M.C.; Malatesta, P.; Ravetti, G.L.; Zona, G.L.; Daga, A.; Corte, G. Sox2 silencing in glioblastoma tumor-initiating cells causes stop of proliferation and loss of tumorigenicity. Stem Cells 2009, 27, 40-48.

25. Hu, T.; Liu, S.; Breiter, D.R.; Wang, F.; Tang, Y.; Sun, S. Octamer 4 small interfering rna results in cancer stem cell-like cell apoptosis. Cancer Res. 2008, 68, 6533-6540.

26. Giangreco, A.; Reynolds, S.D.; Stripp, B.R. Terminal bronchioles harbor a unique airway stem cell population that localizes to the bronchoalveolar duct junction. Am. J. Pathol. 2002, 161, 173-182.

27. Balsara, B.R.; Testa, J.R. Chromosomal imbalances in human lung cancer. Oncogene 2002, 21, 6877-6883.

28. Hussenet, T.; du Manoir, S. Sox2 in squamous cell carcinoma: Amplifying a pleiotropic oncogene along carcinogenesis. Cell Cycle 2010, 9, 1480-1486.

29. Sarkaria, I.; O-charoenrat, P.; Talbot, S.G.; Reddy, P.G.; Ngai, I.; Maghami, E.; Patel, K.N.; Lee, B.; Yonekawa, Y.; Dudas, M.; et al. Squamous cell carcinoma related oncogene/dcun1d1 is highly conserved and activated by amplification in squamous cell carcinomas. Cancer Res. 2006, $66,9437-9444$.

30. Idnurm, A.; Hussenet, T.; Dali, S.; Exinger, J.; Monga, B.; Jost, B.; Dembelé, D.; Martinet, N.; Thibault, C.; Huelsken, J.; et al. Sox2 is an oncogene activated by recurrent 3q26.3 amplifications in human lung squamous cell carcinomas. PLoS One 2010, 5, doi:10.1371/journal.pone.0008960. 
31. Saito, S.; Onuma, Y.; Ito, Y.; Tateno, H.; Toyoda, M.; Hidenori, A.; Nishino, K.; Chikazawa, E.; Fukawatase, Y.; Miyagawa, Y.; et al. Possible linkages between the inner and outer cellular states of human induced pluripotent stem cells. BMC Syst. Biol. 2011, 5 (Suppl 1), doi:10.1186/17520509-5-S1-S17.

32. Adachi, K.; Suemori, H.; Yasuda, S.Y.; Nakatsuji, N.; Kawase, E. Role of sox2 in maintaining pluripotency of human embryonic stem cells. Genes Cells 2010, 15, 455-470.

33. Bernadt, C.T.; Nowling, T.; Rizzino, A. Transcription factor sox-2 inhibits co-activator stimulated transcription. Mol. Reprod. Dev. 2004, 69, 260-267.

34. Huang, P.; Qiu, J.; Li, B.; Hong, J.; Lu, C.; Wang, L.; Wang, J.; Hu, Y.; Jia, W.; Yuan, Y. Role of sox2 and oct4 in predicting survival of hepatocellular carcinoma patients after hepatectomy. Clin. Biochem. 2011, 44, 582-589.

(C) 2012 by the authors; licensee MDPI, Basel, Switzerland. This article is an open access article distributed under the terms and conditions of the Creative Commons Attribution license (http://creativecommons.org/licenses/by/3.0/). 